\title{
A NEW APPROACH TO THE LIMIT THEORY OF RECURRENT MARKOV CHAINS
}

BY

\author{
K. B. ATHREYA AND P. NEY
}

\begin{abstract}
Let $\left\{X_{n} ; n>0\right\}$ be a Harris-recurrent Markov chain on a general state space. It is shown that there is a sequence of random times $\left\{N_{i} ; i>1\right\}$ such that $\left\{X_{N_{i}} ; i>1\right\}$ are independent and identically distributed. This idea is used to show that $\left\{X_{n}\right\}$ is equivalent to a process having a recurrence point, and to develop a regenerative scheme which leads to simple proofs of the ergodic theorem, existence and uniqueness of stationary measures.
\end{abstract}

1. Introduction. The purpose of this paper is to introduce a new tool for the study of the limit theory of a recurrent Markov chain (M.C.) $\left\{X_{n}: n>0\right\}$ on a measurable space $(S, \delta)$. When there is a single point $x_{0}$, called a recurrence point, which is visited infinitely of ten (i.o.) by $\left\{X_{n}\right\}$, a well-known approach to its limit theory is via the imbedded renewal process of returns to $x_{0}$. The key idea in the present work is the observation that a similar regenerative scheme exists for general recurrent chains, in which the role of $x_{0}$ is played by a set which is hit i.o. with the same distribution. In fact, it is possible to adjoin a point $\Delta$ to $S$ and to extend $\left\{X_{n}\right\}$ to $S \cup \Delta$ in such a way that $\Delta$ is a recurrence point for the new process, and that the two processes are equivalent.

We will use this idea to give an elementary proof of the ergodic theorem, and to establish the existence and uniqueness of stationary measures. Extensions to semi-Markov processes and applications to renewal theory will be treated in [1]. Comprehensive background discussions on recurrent chains are available in the books of Doob [3], Neveu [7], Orey [8] and Revuz [9].

2. Recurrence. An irreducible chain having a recurrence point $x_{0}$ is recurrent if it returns to $x_{0}$ with probability one. If such a point does not exist, the notion of recurrence can be captured by requiring that certain sets which are "nonnegligible" with respect to a reference measure are visited "often enough". This idea was introduced by Doeblin [2], in his proof of the ergodic theorem for $\left\{X_{n}\right\}$. He hypothesized that there exist a probability measure $\varphi$ on $(S, \delta)$, numbers $\varepsilon>0, \delta<1$, and an integer $n_{0}<\infty$, such that for all

Received by the editors August 23, 1977 and, in revised form, November 28, 1977.

AMS (MOS) subject classifications (1970). Primary 60J10; Secondary 60K05.

Key words and phrases. Markov chains, regeneration, ergodic theorem, invariant measure. 
$x \in S, E \in \mathcal{S}$

$$
P^{n_{0}}(x, E) \geqslant \varepsilon \quad \text { whenever } \varphi(E) \geqslant \delta .
$$

A slightly stronger version was introduced by Doob [3]. Under such hypotheses it is possible to prove a strong (operator) convergence of $\left\{P^{n}\right\}$ (see e.g. Neveu [7, §5.3], Revuz [9, §6.3]). We will return to this point in \$4.

A weaker condition, introduced by Harris [6], is the so-called $\varphi$-recurrence or Harris-recurrence, which requires the existence of a $\sigma$-finite measure $\varphi$ on $(S, \delta)$ such that $P_{x}\left\{X_{n} \in A\right.$ for some $\left.n\right\}=1$ for all $A \in \delta$ with $\varphi(A)>0$. Under this hypothesis much of the discrete state space theory has been carried over to the general case by Harris [6], Orey [8], and others.

Our regeneration technique becomes particularly transparent if we express recurrence in yet another form.

(2.2) Definition. $\left\{X_{n}\right\}$ is $\left(A, \lambda, \varphi, n_{0}\right)$-recurrent if there exist a set $A \in \delta$, a probability measure $\varphi$ on $A$, a number $0<\lambda<\infty$, and an integer $0<n_{0}$ $<\infty$, such that

(i) $P_{x}\left\{X_{n} \in A\right.$ for some $\left.n \geqslant 1\right\}=1$ for all $x \in S$, and

(ii) $P_{x}\left\{X_{n_{0}} \in E\right\} \geqslant \lambda \varphi(E)$ for all $x \in A$ and $E \subset A$.

RemarKs. (2.3) By making use of the existence of $C$-sets (see Orey [8]) it is easy to show that (2.2) is in fact equivalent to Harris-recurrence.

(2.4) Neveu [7, p. 185] has observed that if (ii) in (2.2) is satisfied with $A=S$, then the Doeblin condition is satisfied.

In this paper we wish to focus on the regeneration idea in its simplest setting, and to that end we will treat only the case $n_{0}=1$ (in (2.2)), in which case we call the chain recurrent and strongly aperiodic. The extension to the general case offers no serious difficulties, and involves typical periodicity and cyclic class arguments. We leave this to a more leisurely exposition of this subject which we are planning, which will also include some applications, discussion of related literature, and some other ramifications. The set $A$ and the measure $\varphi$ are fixed throughout.

3. Regeneration. Our main tool will be the following:

(3.1) REgENERATION LEMMA. If $\left\{X_{n}\right\}$ is recurrent and strongly aperiodic then there exists a random time $N \geqslant 1$ such that $P_{x}(N<\infty)=1$, and for all $x \in S, E \in \mathcal{S}$

$$
P_{x}\left(X_{n} \in E, N=n\right)=\varphi(A \cap E) P_{x}(N=n) .
$$

REMARK. As will be clear from the proof, $N$ is actually defined on a somewhat enlarged state space. It is nonanticipating for $\left\{X_{n}\right\}$ but is not a stopping time for this process.

Here is the idea of the proof. If the chain hits $A$ at a point $x$, at time $k$ randomize the next transition as follows: (i) with probability $p(0<p \leqslant \lambda)$ distribute $X_{k+1}$ over $A$ according to $\varphi$; (ii) with probability $(1-p)$ distribute 
$X_{k+1}$ over the entire state space $S$ according to a transition function $Q(x, \cdot)$, chosen so that the overall transition probabilities for the chain remain unchanged. This is achieved by taking $Q$ so that

$$
P(x, E)=p \varphi(E \cap A)+(1-p) Q(x, E), \quad x \in A, E \in \mathcal{S},
$$

which is possible by (ii) of Definition 2.2. Since $A$ is visited i.o., and each time there is (independent) probability $p>0$ that at the next step " $A$ is entered according to $\varphi$ ", this event will ultimately occur at some time $N<\infty$ a.s. Here is a more formal

Proof OF THE LEMMA. Let $S^{\prime}=S \times\{0,1\}$, $S^{\prime}=$ the $\sigma$-algebra of subsets of $S^{\prime}$ induced by $\mathcal{S}$, and $P^{\prime}$ be a transition function on $S^{\prime} \times \mathcal{S}^{\prime} \rightarrow[0,1]$ defined by

$$
\begin{aligned}
& P^{\prime}\{(x, 0), E \times\{\delta\}\}= \begin{cases}{[p \delta+(1-p)(1-\delta)] P(x, E)} & \text { if } x \notin A, \\
{[p \delta+(1-p)(1-\delta)] Q(x, E)} & \text { if } x \in A,\end{cases} \\
& P^{\prime}\{(x, 1), E \times\{\delta\}\}= \begin{cases}{[p \delta+(1-p)(1-\delta)] P(x, E)} & \text { if } x \notin A, \\
{[p \delta+(1-p)(1-\delta)] \varphi(A \cap E)} & \text { if } x \in A,\end{cases}
\end{aligned}
$$

where $\delta=0$ or 1 and $Q(\cdot, \cdot)$ is as in (3.3). Let $\left\{X_{n}^{\prime}=\left(X_{n}, \delta_{n}\right): n=0,1\right.$, $2, \ldots\}$ be a Markov chain on $\left(S^{\prime}, \delta^{\prime}\right)$ with transition function $P^{\prime}$. Then one can check that

(i) $\left\{X_{n}: n \geqslant 0\right\}$ is an M.C. on $(S, \delta)$ with transition function $P$;

(ii) $\left\{\delta_{n}: n \geqslant 0\right\}$ is a sequence i.i.d. $0-1$ random variables with $P\left(\delta_{n}=1\right)=$ $p$

(iii) $\left\{X_{n}\right\}_{0}^{\infty}$ and $\left\{\delta_{n}\right\}_{0}^{\infty}$ are independent.

Define $N=\inf \left\{n: n \geqslant 1, X_{n-1}^{\prime} \in A x\{1\}\right\},=\infty$ if $\{-\}$ is empty. It remains only to observe that $P_{(x, \delta)}^{\prime}\{N<\infty\}=1$. To see this notice that

$$
\begin{aligned}
P_{(x, \delta)}^{\prime}(N=\infty) & =P_{(x, \delta)}^{\prime}\left(N=\infty, X_{n} \in A \text { for } \infty \text { many } n\right) \\
& =P_{(x, \delta)}^{\prime}\left(\delta_{L_{i}}=0 \text { for all } i\right),
\end{aligned}
$$

where $L_{1}, L_{2}, \ldots$ are the successive hitting times of $A$ by $\left\{X_{n}\right\}$. The last probability $=0$ due to the independence of $\left\{X_{n}\right\}$ and $\left\{\delta_{n}\right\}$.

REMARK. Note that the random time $N$ is a stopping time for $\left\{X_{n}^{\prime}\right\}$.

We recall that $X_{N}$ is distributed over $A$ according to $\varphi$. Reapplying the regeneration lemma, there exists a regeneration time $M$ for $\left\{X_{n}\right\}$ initiated with $X_{0}=X_{N}$, i.e. with distribution $\varphi$. Let $N=N_{1}, N+M=N_{2}$. Continuing in this way, we use the lemma to observe that under $(A, \lambda, \varphi, 1)$ recurrence we have

(3.4) Corollary. There exists a sequence of random times $N_{1}, N_{2}, \ldots$ for $X_{n}$ such that $P_{x}\left(N_{k}<\infty\right) \equiv 1$ for all $k \geqslant 1$, and

$$
P_{x}\left(X_{n} \in E, N_{k}=n\right)=\varphi(E \cap A) P_{x}\left(N_{k}=n\right) .
$$


Here $P_{x}\left(N_{1}=n\right)=P_{x}(N=n)$ as in (3.2), while for $k \geqslant 2, N_{k}=N_{1}+M_{2}$ $+\cdots+M_{k}$, where the $M_{i}$ 's are i.i.d. (and independent of $\left.N_{1}\right)$, and $P_{x}\left(M_{i}=\right.$ $n)=P_{\varphi}(N=n), x \in S, n \geqslant 1$.

Thus $\left\{X_{N_{i}}: i \geqslant 1\right\}$ are independently, identically distributed according to $\varphi$, and in this sense the process regenerates itself at $\left\{N_{i}\right\}$.

There is some similarity between the kind of randomization (or smoothing) which goes on in our lemma, and a coupling technique for MC.'s which goes back to Doeblin [2], and has been developed by Griffeath [5].

4. Ergodicity. Let $\mathscr{B}$ denote the Banach space of bounded measurable functions $f: S \rightarrow R$, with sup norm $\|f\|$. If $\mu$ is a finite measure on $(S, \delta)$ and $f \in \mathscr{B}$, let $\mu f=\int f(x) \mu(d x),(\mu P)(x)=\int \mu(d s) P(s, x),\|\mu\|=$ total variation norm of $\mu . P^{n}$ is defined as an operator on $\mathscr{B}$ by the formula $\left(P^{n} f\right)(x)=$ $E_{x} f\left(X_{n}\right)$. Let $\||P|||$ denote its norm. Let $T_{B}$ denote the first hitting time of any set $B \in \mathcal{S}$ by $\left\{X_{n}\right\} ; N=$ the random time in Lemma (3.1).

(4.1) THEOREM. (i) If $\left\{X_{n}\right\}$ is recurrent and strongly aperiodic, with invariant probability measure $\pi(\cdot)$, then for all $x \in S$

$$
\left\|P^{n}(x, \cdot)-\pi(\cdot)\right\| \rightarrow 0 \text { as } n \rightarrow \infty .
$$

(ii) If furthermore $\sup _{x} P_{x}\left(T_{A}>t\right)<1$ for some $t<\infty$, then $\left\|P^{n}-\pi\right\| \rightarrow$ 0 .

Proof OF (i). Let $v_{n}=E_{\varphi} f\left(X_{n}\right), a_{n}=E_{\varphi}\left\{f\left(X_{n}\right) ; N>n\right\}, p_{n}=P_{\varphi}(N=n)$, $m=E_{\varphi} N$. The condition $n_{0}=1$ implies that $\left\{p_{n}\right\}$ is aperiodic, and hence by the renewal theorem $u_{n} \equiv \Sigma_{k} p_{n}^{* k} \rightarrow m^{-1}$ as $n \rightarrow \infty$. But by the regeneration lemma, $v_{n}$ satisfies the renewal equation $v_{n}=a_{n}+\sum_{j=0}^{n} p_{j} v_{n-j}$, and hence

$$
v_{n}=\sum_{j=0}^{n} a_{n-j} u_{j} \rightarrow m^{-1} \sum_{0}^{\infty} a_{k}
$$

provided $\Sigma\left|a_{k}\right|<\infty$. To see this let $N=N_{1}, N_{2}, \ldots$ denote the regeneration times, and $K_{n}=\sup \left\{k: N_{k} \leqslant n\right\}$. Since $\left(S_{K_{n}} / K_{n}\right)<\left(n / K_{n}\right)<\left(S_{K_{n}+1} / K_{n}\right)$, the law of large numbers implies that $K_{n} / n \rightarrow\left(E_{\varphi} N\right)^{-1}$ a.s., and since $0 \leqslant K_{n} / n \leqslant 1, E_{\mu}\left(K_{n} / n\right) \rightarrow\left(E_{\varphi} N\right)^{-1}$ for any initial distribution $\mu(\cdot)$. Now, $K_{n}$ can be written as $\sum_{i=1}^{n} \delta_{i}$, where $\delta_{i}=1$ or 0 depending on whether a regeneration occurs at $i$ or not. Since $P_{\pi}\left(\delta_{i}=1\right)=p P_{\pi}\left(X_{i-1} \in A\right)=p \pi(A)$, $E_{\pi} K_{n}=n p \pi(A)$. Furthermore the fact that $P_{x}\left(X_{n} \in A\right.$ for some $\left.n>1\right) \equiv 1$ implies that $P_{\pi}\left(X_{n} \in A\right.$ for some $\left.n \geqslant 1\right)=1$, and since $P_{\pi}\left(X_{n} \in A\right) \equiv \pi(A)$ for all $n$ by stationarity, we see that $\pi(A)$ has to be nonzero. Thus, $E_{\varphi}(N)=$ $(p \pi(A))^{-1}<\infty$. Thus also $\Sigma\left|a_{n}\right|<\|f\| \Sigma_{n} P_{\varphi}(N>n)<\infty$, proving (4.2).

Now let $x$ be any initial state. Since lim $v_{n}$ exists and

$$
E_{x} f\left(X_{n}\right)=E_{x}\left\{f\left(X_{n}\right) ; N>n\right\}+E_{x}\left\{v_{n-N} ; N<n\right\},
$$


we see that $\lim E_{x} f\left(X_{n}\right)$ exists, and hence by the bounded convergence theorem $\lim E_{\mu} f\left(X_{n}\right)=\lim v_{n}$ for any initial distribution $\mu$. Choosing $\mu$ to be $\pi$, we conclude that

$$
E_{x} f\left(X_{n}\right) \rightarrow m^{-1} \sum a_{k}=\pi f \text { for all } x \in S .
$$

We can strengthen the convergence to total variation norm. Let $\varepsilon_{K}=$ $\sup \left\{\left|u_{j}-m^{-1}\right|: j>K\right\}$, and note that $0<u_{n}<1$ and $0<m^{-1} \leqslant 1$. Thus

$$
\begin{aligned}
\left|\left(\varphi P^{n}-\pi\right) f\right| & =\left|v_{n}-\pi f\right|<\sum_{0}^{n}\left|a_{n-j}\right| \cdot\left|u_{j}-m^{-1}\right|+m^{-1} \sum_{j>n}\left|a_{j}\right| \\
& <\sum_{j>n-K}\left|a_{j}\right|+\varepsilon_{K} \sum_{j<n-K}\left|a_{j}\right|<\|f\|\left\{\varepsilon_{K}+\sum_{j>n-K} P_{\varphi}(N>j)\right\} .
\end{aligned}
$$

Let $\gamma_{n}=\sup \left\{\left|v_{n}-\pi f\right| /\|f\|: 0<\|f\|<\infty\right\}=\left\|\varphi P^{n}-\pi\right\|$. Then

$$
\gamma_{n}=\varepsilon_{K}+\sum_{j>n-K} P_{\varphi}(N>j)
$$

and taking $K$ large we see that $\gamma_{n} \rightarrow 0$ as $n \rightarrow \infty$. Finally use (4.3) to observe that

$$
\left|E_{x} f\left(X_{n}\right)-\pi f\right| /\|f\|<2 P_{x}(N>n)+E_{x}\left\{\left|v_{n-N}-\pi f\right| /\|f\| ; N<n\right\} .
$$

Hence $\left\|P^{n}(x, \cdot)-\pi(\cdot)\right\|<2 P_{x}(N>n)+E_{x}\left\{\gamma_{n-N} ; N<n\right\}$, and the bounded convergence theorem then implies (i).

Proof of (ii). Suppose first that $\sup _{x} P_{x}(N>n) \rightarrow 0$ as $n \rightarrow \infty$, and for a given $\varepsilon$, choose $n_{0}$ so that $\sup _{x} P_{x}(N>n)<\varepsilon$ and $\gamma_{n}<\varepsilon$ for $n>n_{0}$. Let $\left|v_{n}-\pi f\right| /\|f\|=\xi_{n}(f)$. Then for $n>2 n_{0}$,

$$
\begin{aligned}
\sup _{x, f} E_{x}\left\{\xi_{n-N}(f) ; N \leqslant n\right\}< & \sup E_{x}\left\{\xi_{n-N}(f) ; N<n-n_{0}\right\} \\
& +\sup E_{x}\left\{\xi_{n-N}(f) ; n-n_{0}<N \leqslant N\right\} \\
& <\sup _{n>n_{0}} \gamma_{n}+\sup _{x} P_{x}\left(N>n_{0}\right)<2 \varepsilon,
\end{aligned}
$$

and hence (by (4.7))

$$
\begin{aligned}
& \sup _{x, f}\left\{\left|E_{x} f\left(X_{n}\right)-\pi f\right| /\|f\|\right\} \\
& \quad<2 \sup _{x} P_{x}(N>n)+\sup _{x, f} E_{x}\left\{\xi_{n-N}(f) ; N<n\right\}<4 \varepsilon .
\end{aligned}
$$

Thus $\left\|P^{n}-\pi \mid\right\| \rightarrow 0$ as $n \rightarrow \infty$. It remains only to observe that the hypothesis of (ii) implies $\sup _{x} P_{x}(N>n) \rightarrow 0$. To see this, pick $n_{1}$ so that $\sup _{x} P_{x}\left(T_{A}\right.$ $\left.>n_{1}\right)<1-\eta$ for some $\eta>0$. Then 


$$
\begin{aligned}
\delta_{n} & \equiv \sup _{x} P_{x}\left(N>n n_{1}\right) \\
& \leqslant \sup _{x} P_{x}\left\{\sum_{i=1}^{n n_{1}} \chi_{A}\left(X_{i}\right)>k_{n}, N>n n_{1}\right\}+\sup _{x} P_{x}\left\{\sum_{i=1}^{n n_{1}} \chi_{A}\left(X_{i}\right)<k_{n}\right\} \\
& \leqslant(1-p)^{k_{n}}+P\left\{B_{n, \eta} \leqslant k_{n}\right\},
\end{aligned}
$$

where $B_{n, \eta}$ is binomial $(n, \eta)$. Now take $k_{n}=\varepsilon n$, with $\varepsilon$ small enough so that $\delta_{n} \rightarrow 0$.

REMARK. Note that actually $\delta_{n} \rightarrow 0$ geometrically fast, and hence so does $\left|u_{n}-m^{-1}\right| \rightarrow 0$ (Stone [10]). Thus the convergence $E_{x} f\left(X_{n}\right) \rightarrow \pi f$ is also geometric.

When an invariant probability measure does not exist, then one still has

(4.8) THEOREM (OREY). If $\left\{X_{n}\right\}$ is recurrent and strongly aperiodic then for any two initial distributions $\mu$ and $\eta$,

$$
\left\|\mu P^{n}-\nu P^{n}\right\| \rightarrow 0 \text {. }
$$

Proof. Let $f: S \times N^{+} \rightarrow R$ be a bounded space-time harmonic function for $\left\{X_{n}\right\}$, i.e. $f(s, m)=E\left\{f\left(X_{1}, m+1\right) \mid X_{0}=s\right\}$. Then $Y_{n}=f\left(X_{n}, m+n\right)$, $n>0$, is a bounded martingale and letting $g(n)=E_{\varphi} f\left(X_{0}, n\right)$, and $N$ be as before,

$$
\begin{aligned}
f(s, m) & =E_{s} Y_{0}=E_{s} f\left(X_{N}, m+N\right) \\
& =\sum g(m+k) P_{s}(N=k)=E_{s} g(m+N) .
\end{aligned}
$$

Integrating with respect to $\varphi$ we get $g(m)=E_{\varphi} g(m+N)$. Since $g(\cdot)$ is bounded and $N$ is aperiodic this implies that $g$ is constant (see Feller [4, p. 351]), and hence by (4.9) $f$ is constant. Orey [8, Proposition 4.3] has shown that this in turn implies (4.9).

5. Equivalence to a chain with a recurrence point. Adjoin a point $\Delta$ to $S$, and extend $\left\{X_{n}\right\}$ to a M.C. $\left\{\tilde{X}_{n}\right\}$ on $S \cup \Delta$, with transition function $\tilde{P}$ defined by

$$
\tilde{P}(x, E)= \begin{cases}P(x, E-\Delta) & \text { for } x \notin A \cup \Delta, \\ (1-p) Q(x, E-\Delta)+p \chi_{E}(\Delta) & \text { for } x \in A, \\ (1-p) \int_{A} Q(z, E-\Delta) \varphi(d z)+p \chi_{E}(\Delta) & \text { for } x=\Delta .\end{cases}
$$

Note that $\Delta$ is a recurrence point since $\left\{\tilde{X}_{n}\right\}$ is in $A$ i.o., and $\tilde{P}(x, \Delta)=p>0$ for $x \in A$. For bounded measurable $f: S \rightarrow R$ define

$$
\begin{aligned}
& \tilde{f}(x)=f(x) \quad \text { for } x \in S, \\
& \tilde{f}(\Delta)=\int_{A} f(z) \varphi(d z)=E f\left(X_{N}\right) .
\end{aligned}
$$

If $\pi(\cdot)$ is a measure on $S$, define the measure $\tilde{\pi}(\cdot)$ on $S \cup \Delta$ by 


$$
\begin{aligned}
\tilde{\pi}(E) & =\pi(E)-p \pi(A) \varphi(A \cap E), \quad E \subset S, \\
\tilde{\pi}(\Delta) & =p \pi(A) .
\end{aligned}
$$

(5.4) EquivalenCE THeOREM. (i) Let $\left\{X_{n}\right\}$ be recurrent and strongly aperiodic, and $\left\{\tilde{X}_{n}\right\}$ be an M.C. with transition function $\tilde{P}$. Then for any initial distribution $\mu$ on $S$

$$
E_{\mu} f\left(X_{n}\right)=E_{\mu} \tilde{f}\left(X_{n}\right) .
$$

(ii) If $\pi$ is a stationary measure for $P$, then $\tilde{\pi}$ is a stationary measure for $P$, and conversely. If either $\pi$ or $\tilde{\pi}$ is unique (up to multiplicative constants), then so is the other. If $\pi$ or $\tilde{\pi}$ is a probability measure, so is the other.

The proof is a straightforward verification of definitions and formulas, so we will leave it to the reader.

6. Invariant measures. Harris-recurrent chains are known to have unique, $\sigma$-finite, invariant measures (Harris [6], Orey [8]). (Uniqueness will always be understood to be up to a multiplicative constant.) The proofs have been quite involved, but our regeneration scheme now provides an easy alternative.

(6.1) THEOREM. A recurrent, strongly aperiodic M.C. $\left\{X_{n}\right\}$ has a unique invariant measure. It is finite if and only if $E_{\varphi} N<\infty$.

Proof. By part (ii) of the Equivalence Theorem (5.4) it is sufficient to prove the theorem when $\left\{X_{n}\right\}$ has a recurrence point (say $x_{0}$ ). Note that in this case

$$
\bigcup_{n}\left\{x: P^{n}\left(x, x_{0}\right)>0\right\}=S .
$$

Let $N=\inf \left\{n: n \geqslant 1, X_{n}=x_{0}\right\}$, and define

$$
\nu(E)=E_{x_{0}} \sum_{i=0}^{N-1} \chi_{E}\left(X_{i}\right), \quad E \in \delta
$$

Clearly $\nu(\cdot)$ is finite iff $E_{\varphi} N<\infty$.

(i) $\nu(\cdot)$ is an invariant measure. To see this write

$$
\begin{aligned}
\nu(E) & =\chi_{E}\left(x_{0}\right)+\sum_{i=1}^{\infty} E_{x_{0}}\left\{\chi_{E}\left(X_{i} ; N>i-1\right)\right\}-\sum_{i=1}^{\infty} E_{x_{0}}\left\{\chi_{E}\left(X_{i}\right) ; N=i\right\} \\
& =\chi_{E}\left(x_{0}\right)+E_{x_{0}}\left\{\sum_{i=1}^{\infty} P\left(X_{i-1}, E\right) \chi_{(N>i-1)}\right\}-E_{x_{0}} \chi_{E}\left(X_{N}\right) .
\end{aligned}
$$

The first and last terms cancel, and the middle term

$$
=E_{x_{0}} \sum_{i=0}^{N-1} P\left(X_{i}, E\right)=\int \nu(d x) P(x, E) .
$$

(ii) $\nu(\cdot)$ is unique (up to multiplicative constants). Suppose that $\lambda(\cdot)$ is 
another invariant measure. Then

$$
\begin{aligned}
\lambda(E)= & \int_{S} \lambda(d x) P(x, E)=\lambda\left(x_{0}\right) P\left(x_{0}, E\right)+\int_{S-x_{0}} \lambda(d x) P(x, E) \\
= & \lambda\left(x_{0}\right) P_{x_{0}}\left(X_{1} \in E\right)+\lambda\left(x_{0}\right) P_{x_{0}}\left(X_{1} \neq x_{0}, X_{2} \in E\right) \\
& +\int_{S-x_{0}} \int_{S-x_{0}} \lambda(d z) P(z, d x) P(x, E) .
\end{aligned}
$$

Iterating this relation, we get for all $n>1$,

$$
\begin{aligned}
\lambda(E)>\lambda\left(x_{0}\right)\{ & P_{x_{0}}\left(X_{1} \in E\right)+P_{x_{0}}\left(X_{1} \neq x_{0}, X_{2} \in E\right)+\ldots \\
& \left.+P_{x_{0}}\left(X_{1} \neq x_{0}, \ldots, X_{n-1} \neq x_{0}, X_{n} \in E\right)\right\} .
\end{aligned}
$$

Letting $n \rightarrow \infty$ and observing that $X_{N}=x_{0}$, yields

$$
\lambda(E)>\lambda\left(x_{0}\right) \sum_{i=1}^{\infty} E_{x_{0}}\left\{\chi_{E}\left(X_{i}\right) ; N>i\right\}=\lambda\left(x_{0}\right) E_{x_{0}} \sum_{i=1}^{N} \chi_{E}\left(X_{i}\right)=\lambda\left(x_{0}\right) \nu(E) \text {. }
$$

Thus, since $\nu\left(x_{0}\right)=1$ by definition of $\nu(\cdot)$,

$$
\lambda\left(x_{0}\right)=\int_{S} \lambda(d x) P^{n}\left(x, x_{0}\right) \geqslant \lambda\left(x_{0}\right) \int \nu(d x) P^{n}\left(x, x_{0}\right)=\lambda\left(x_{0}\right) \nu\left(x_{0}\right)=\lambda\left(x_{0}\right)
$$

and hence

$$
\int_{S} \lambda(d x) P^{n}\left(x, x_{0}\right)=\lambda\left(x_{0}\right) \int_{S} \nu(d x) P^{n}\left(x, x_{0}\right) .
$$

Let $D_{n}=\left\{x: P^{n}\left(x, x_{0}\right)>0\right\}$. To complete the proof it is sufficient (by (6.2)) to show that

$$
\lambda(E)=\lambda\left(x_{0}\right) \nu(E) \quad \text { for all } E \subset D_{n}, \quad n>1 .
$$

If this is false, then by (6.4) there must be an $E_{0} \subset D_{n}$ for some $n<\infty$ such that $\lambda\left(E_{0}\right)>\lambda\left(x_{0}\right) \nu\left(E_{0}\right)$. But then

$$
\int_{E_{0}} P^{n}\left(x, x_{0}\right) \lambda(d x)>\lambda\left(x_{0}\right) \int_{E_{0}} P^{n}\left(x, x_{0}\right) \nu(d x),
$$

while

$$
\int_{S-E_{0}} P^{n}\left(x, x_{0}\right) \lambda(d x)>\lambda\left(x_{0}\right) \int_{S-E_{0}} P^{n}\left(x, x_{0}\right) \nu(d x) .
$$

This contradicts (6.5).

ADDENDUM. We have recently learned of concurrent work along similar lines by E. Nummelin, Helsinki University of Technology.

\section{REFERENCES}

1. K. B. Athreya, D. McDonald and P. E. Ney, Limit theorems for semi-Markov processes and renewal theory for Markov chains, Ann. Probability (to appear). 
2. W. Doeblin, Éléments d'une théorie générale des chaines simples constantes de Markoff, Ann. Sci. Ecole Norm. Sup. 57 (1940), 61-111.

3. J. L. Doob, Stochastic processes, Wiley, New York, 1953.

4. W. Feller, An introduction to probability theory and its applications, Vol. 2, Wiley, New York, 1966.

5. D. Griffeath, Coupling methods for Markov processes, Thesis, Cornell University, 1976; Advances in Math. (to appear).

6. T. E. Harris, The existence of stationary measures for certain Markov processes, Proc. Third Berkeley Symposium on Mathematical Statistics and Probability, 1954-1955, Vol. II, Univ. of California Press, Berkeley and Los Angeles, pp. 113-124.

7. J. Neveu, Mathematical foundations of the calculus of probability, Holden-Day, San Francisco, 1965.

8. S. Orey, Limit theorems for Markov chain transition probabilities, Van Nostrand, New York, 1971.

9. D. Revuz, Markov chains, North-Holland, Amsterdam, 1975.

10. C. Stone, On moment generating functions and renewal theory, Ann. Math. Statist. 36 (1965), 1298-1301.

Department of Applied Mathiematics, Indian Institute of Science, Bangalore 560012, INDIA

Department of Mathematics, University of Wisconsin, Madison, Wisconsin 53706 